Case report

\title{
Calcifying nested stromal-epithelial tumor (CNSET) of the liver
}

\author{
Victor lanole ${ }^{1}$, Loredana Beatrice Ungureanu ${ }^{*}{ }^{1}$, Eugenia Moroșan ${ }^{1}$, Cristian Dumitru \\ Lupașcu $^{1,2}$
}

1 "Grigore T. Popa" University of Medicine and Pharmacy, lasi, Romania; ${ }^{2}$ Department of Surgery, "Sf. Spiridon" University Emergency Hospital, lasi, Romania

\begin{abstract}
Calcifying nested stromal-epithelial tumor (CNSET) of the liver is an extremely rare primary tumor of nonhepatic and non-biliary origins which generally affects children and young adults, mainly women. We report a case of 28-year-old woman with a history of oral contraceptive consumption for 9 years, who underwent surgery for a large right hepatic mass that proved to be a CNSET, according to the pathology report. Postoperatively the patient had a slow recovery and after discharge the patient was sent to the oncology department where she underwent several treatment cycles with Imatinib. The case report documented all the clinical and histopathological features necessary for diagnosis of CNSET. CNSET of liver should always be considered and included in the differential diagnosis of hepatic tumors especially if the patient is a young woman with a long history of oral contraceptive consumption and an abdominal palpable mass.
\end{abstract}

Keywords: calcifying nested stromal-epithelial tumor; hepatic tumor; hepatoblastoma; liver; mixed tumor

\section{Introduction}

Calcifying nested stromal-epithelial tumor (CNSET) of the liver is an extremely rare primary tumor of non-hepatic and non-biliary origins [1] consisting of nests of spindled and epithelioid cells that can associate calcification or bone formation [2]. As far as we know only 37 cases were previously described in the literature under different names like: desmoplastic nested spindle cell tumor of the liver, ossifying malignant mixed epithelial and stromal tumor of the liver [3], nested stromal epithelial tumor of the liver and ossifying stromal-epithelial tumor [4]. It was first

Received: February 2018; Accepted after review: March 2018; Published: March 2018.

*Corresponding author: Loredana Ungureanu, MD, PhD, Pathology Department, "Grigore T. Popa" University of Medicine and Pharmacy lasi, 16 Universitatii Street, 700115, Iasi, Romania

Email: loredana.ungureanu81@gmail.com described by Ishak et al. in 2001 [5] and the term CNSET was proposed by Makhlouf et al. in 2009 [4]. It generally affects children and young adults, mainly women and it is frequently located in the right hepatic lobe [5].

\section{Case report \\ Preoperative assessment and surgical therapy \\ A 28-year-old Caucasian woman was} admitted to Surgery Department of our hospital with upper abdominal pain, asthenia and fatigue. The patient had a history of oral contraceptive consumption for 9 years, but no history of hepatitis, alcohol use, and consumption of hepatotoxic medication or a family history of hepatic malignancy.

Physical examination revealed pallor, pain in the upper abdominal area and a large palpable mass in the right hypochondriac and lumbar region. No Cushingoid or other clinical symptoms were present. 
Laboratory tests revealed a low level of hemoglobin $(9.4 \mathrm{~g} / \mathrm{dl})$ and hematocrit $(29.9 \%)$. Serum levels of gamma-glutamyl transferase, aspartate transaminase, alanine transaminase, alpha-fetoprotein, carbohydrate antigen 19-9 and carcinoembryonic antigen were normal. Serology results for hepatitis B and $C$ were negative.

Abdominal ultrasound and computer tomography (CT) revealed a large nonhomogeneous mass with irregular borders,
Doppler signal and small nodular calcifications that involved the right hepatic lobe entirely (Figure 1). The CT images showed a mass confined to the liver that caused mass effect and compression to the adjacent structures. No biopsy was taken.

Based on the preoperative assessment the patient was scheduled for liver resection, with a presumptive diagnosis of hepatocellular carcinoma. Exploratory laparotomy was done followed by a right hepatectomy.
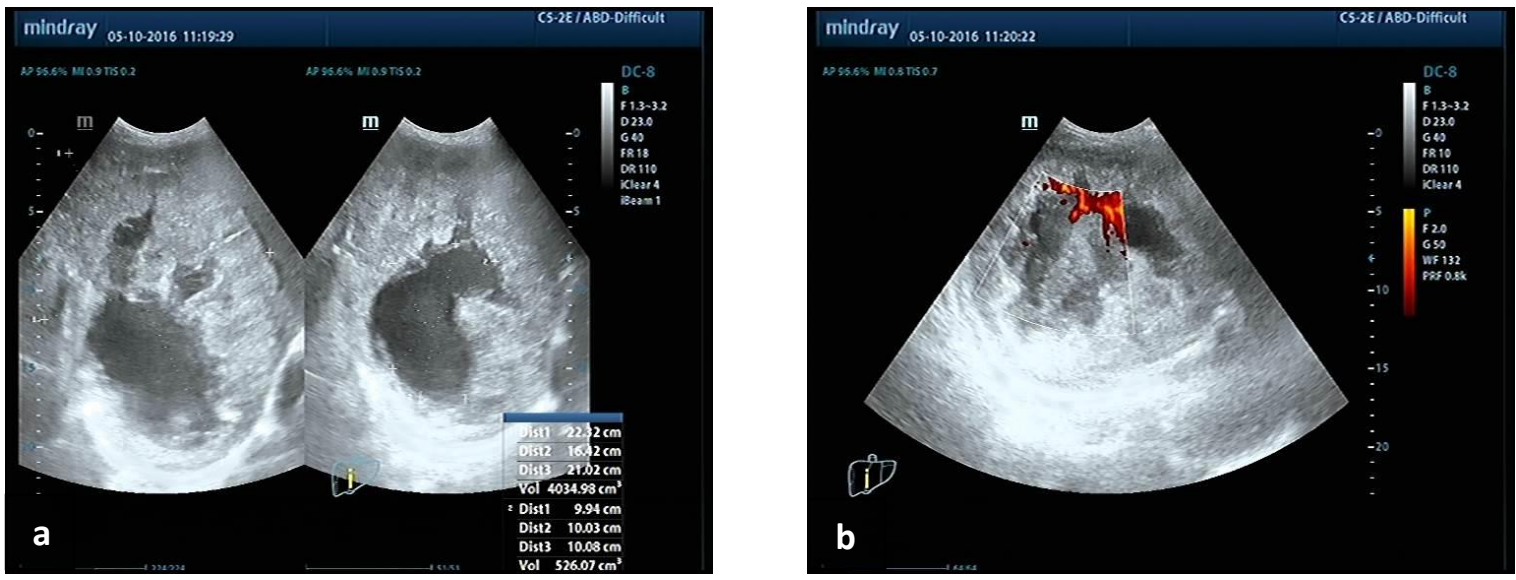

Fig. 1. Ultrasound shows a large nonhomogeneous mass with irregular borders (a) and Doppler signal present (b).

\section{Pathology - gross appearance}

The tumor had a non-encapsulated, still well-defined, lobulated feature. It appeared as a large mass of about $34 / 19 / 12 \mathrm{~cm}$ that occupied the entire right hepatic lobe. On cut surface, the tumor was solid, heterogeneous, with white areas and large cystic spaces (Figure 2).
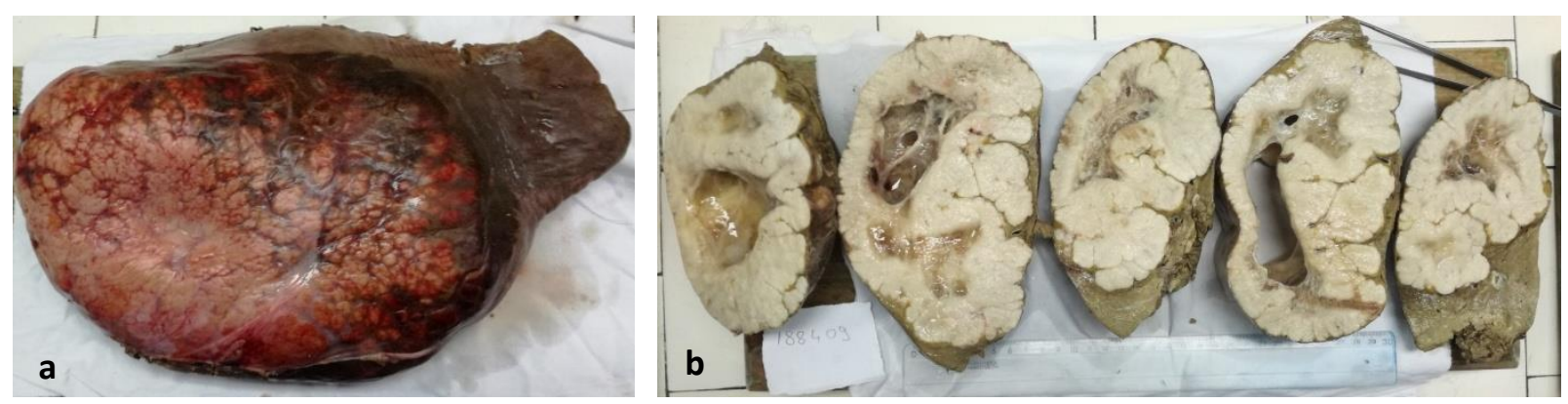

Fig. 2. Gross image showed a relative well defined non-encapsulated mass (a) with a lobulated, white, solid and cystic appearance on cut surface (b)

\section{Pathology - microscopical and immunohistochemical analysis}

Histology revealed a multinodular, lobulated, non-encapsulated tumor composed from nests of spindle and epithelioid cells with small, central calcifications, surrounded by moderate fibrous hypercellular stroma including blood vessels. Tumor cells had irregular shape, scant cytoplasm, oval, hyperchromatic or vesicular nuclei and low nuclear atypia (Figure 3). The mitotic count was low (1-2 mitosis/10 HPF). 


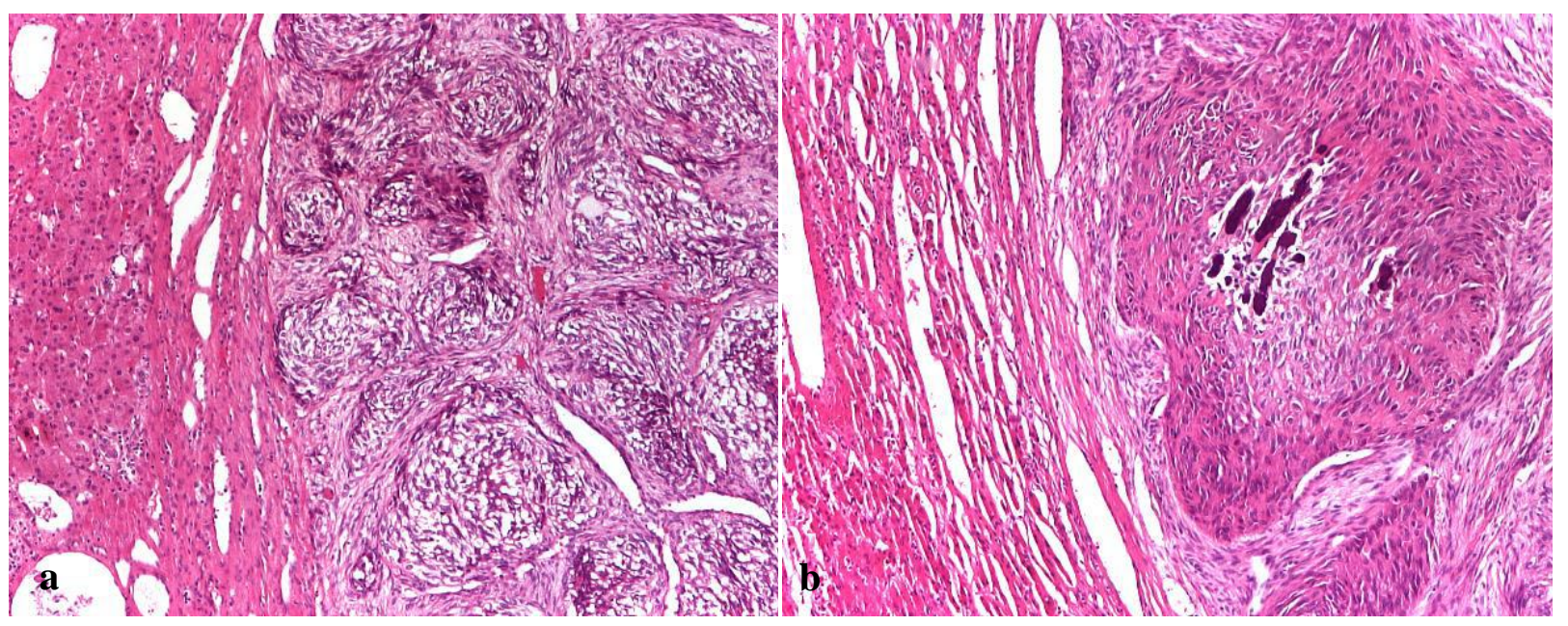

Fig. 3. Microscopical features of CNSET: a. lobular non-encapsulated tumor, composed of nests of spindle and epithelioid cells, surrounded by a fibrous hypercellular stroma with blood vessels (HE, x40); b. central calcifications of nests $(\mathrm{HE}, \mathrm{x} 40)$

Due to the unusual histological appearance, the immunohistochemical (IHC) tests were done in a stepwise fashion, in order to confirm and/or to exclude certain presumable entities.

Therefore, the panel of markers included CD117, DOG1 and CD34 for gastrointestinal stromal tumor (GIST), CK AE1/AE3, CK5, CK7, CK20, TTF-1 and p63 for carcinoma of digestive, pulmonary or squamous origin, Synaptophysin and Chromogranin A for neuroendocrine neoplasia, S100, Melan-A and HMB45 for malignant melanoma, HSA for hepatocellular carcinoma or hepatoblastoma, Vimentin and SMA for sarcoma and Ki-67 to evaluate the mitotic activity.

Tumor cells were positive for CK AE1/AE3, S100, focally positive for CD117 and Vimentin, while negative for the rest of the markers. Ki-67 showed positivity in less than $10 \%$ of tumor cells.

A rare and lesser known entity, CNSET, had to be proven using CD56, WT1 and EMA. Tumor cells were positive for CD56, focally positive for WT1 and negative for EMA, a pattern consistent with the diagnosis of CNSET (Figure 4).

\section{Postoperative assessment}

Postoperatively the patient had a slow recovery with a small fluid collection in right hypochondriac region, right lumbar region and hypogastrium that resolved in the following weeks. After discharge the patient was sent to oncology department to continue the treatment with several chemotherapy cycles of Imatinib.

\section{Discussion}

Calcifying nested stromal-epithelial tumor (CNSET) of the liver is an extremely rare tumor occurring mainly in females $(76 \%)$ with nearly $70 \%$ of reported cases in children [5]. This rare entity typically displays nests of spindled and epithelioid cells that associate calcifications or bone formation, surrounded by moderate fibrovascular stoma [1-7].

The small number of CNSETs reported in literature makes it challenging to properly evaluate the clinical behavior and outcome in patients with this type of tumors [1, 8]. So far, only three metastatic cases have been reported (lung, liver and lymph nodes) and a few cases had recurrences, features which confirm that CNSET could be considered a low-grade malignancy $[3,5,9]$.

The pathogenesis of CNSET remains uncertain. A few cases had a Cushing syndrome that resolved after surgery $[3,8]$. Three cases were reported in patients with Beckwith-Weideman syndrome [1]. Similar to our case, a few others had a history of oral contraceptive pills consumption but no direct association was proven [5]. 
Other theories suggest the implication of WT1, due to its role in the transformation of mesenchymal cells into epithelial cells [5] and of Beta-catenin and other mesenchymalepithelial transition factors like: SNAIL, SLUG, TWIST, c-Met, vimentin and E-cadherin. Apart from E-cadherin, all others have an increased expression in the CNSET tumor cells. This hypothesis needs further research and validation due to the small number of cases included in the study [10].

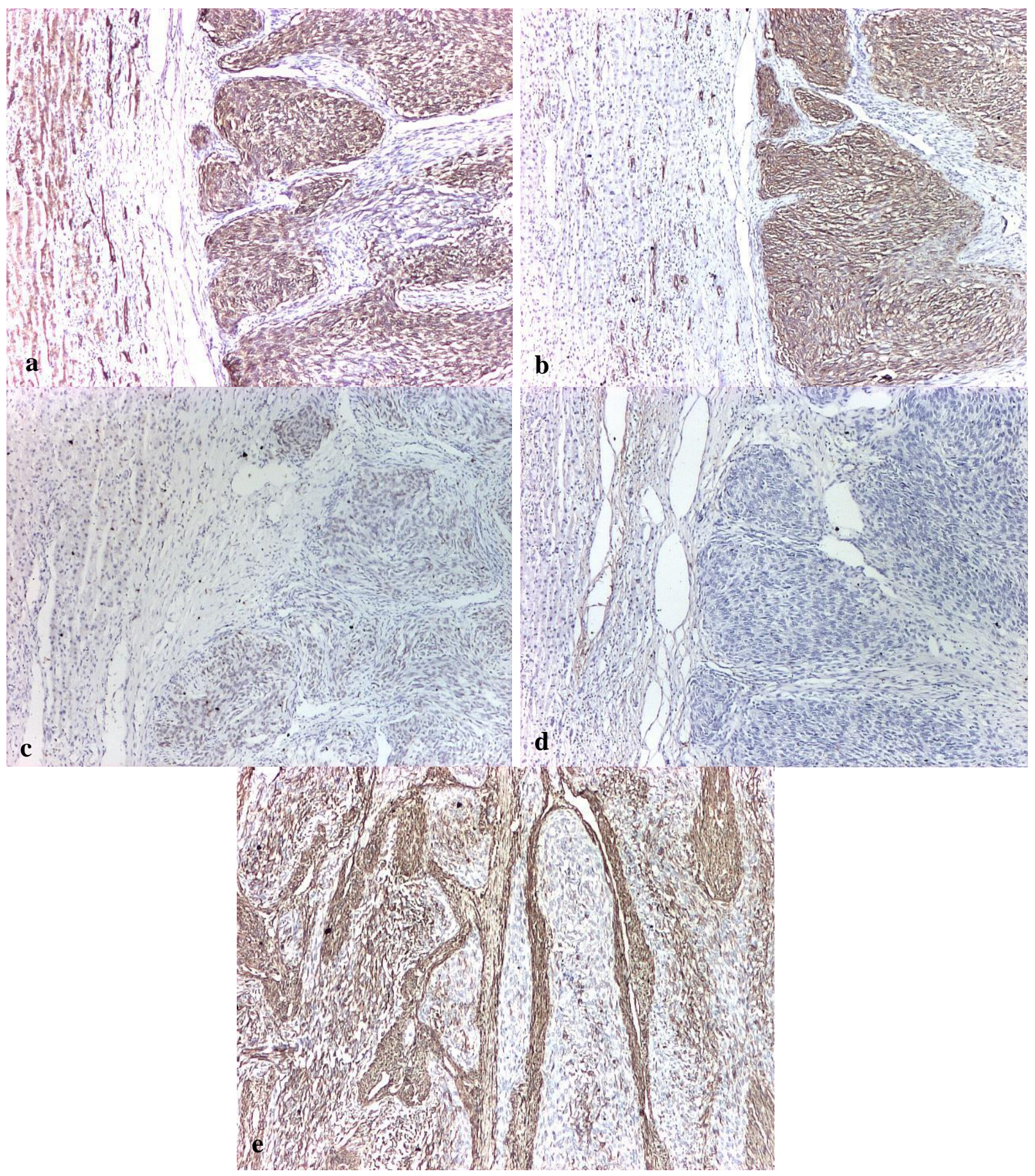

Fig. 4. IHC profile of CNSET: a. strong CK AE1/AE3 positivity, cytoplasmic staining of tumor cells (anti-AE1/AE3 $\mathrm{Ab}, \mathrm{x} 40$ ); b. strong CD 56 positivity, membrane staining of tumor cells (anti-CD $56 \mathrm{Ab}, \mathrm{x} 40$ ); c. weak WT1 positivity, nuclear staining of tumor cells (anti-WT1 Ab, x40); d. negative EMA, membrane staining of tumor cells (anti-EMA Ab, x40); e. strong Vimentin positivity, cytoplasmic staining of spindle cells (anti-Vimentin Ab, x40). 
The differential diagnosis of this rare entity includes desmoplastic small round cell tumor (DSRCT), synovial sarcoma, hepatoblastoma and endocrine tumors $[4,6]$. Opposite to CNSET, DSRCT can be found mainly in adolescent males and is highly aggressive, lacks the fibrovascular stroma and the myofibroblastic collars [4, 6, 7]. On IHC, DSRCT is positive for desmin and the molecular biology shows a ESW1 to WT1 fusion characteristic for this tumor [4]. The presence of a haemangiopericytic vascular pattern and the positivity for CD99 are in favor of synovial sarcoma, although cases with osteoid formation and calcification have been described [4, 6, 11]. Hepatoblastoma, particularly mixed, epithelial and mesenchymal subtypes are rarely seen in adults and are characterized by fetal and/or embryonal hepatocyte differentiation components. It lacks the typical CNSET stroma pattern and it is positive for HepPar-1 and AFP [4, 7]. CNSET can be differentiated from endocrine tumors by the stroma pattern, positivity for CD56 and the negativity for neuroendocrine markers [6].

\section{References}

1. Khoshnam $\mathrm{N}$, Robinson $\mathrm{H}$, Clay $\mathrm{MR}$, et al. Calcifying nested stromal-epithelial tumor (CNSET) of the liver in Beckwith-Wiedemann syndrome. Eur J Med Genet 2017; 60(2):136139.

2. Hamilton SR, Aaltonen LA (Eds): World Health Organization Classification of Tumours. Pathology and Genetics of Tumours of the Digestive System. IARC Press: Lyon 2000.

3. Weeda VB, de Reuver PR, Bras $\mathrm{H}$ et al. Cushing syndrome as presenting symptom of calcifying nested stromal-epithelial tumor of the liver in an adolescent boy: a case report. $J$ Med Case Rep 2016; 10:160.

4. Makhlouf HR, Abdul-Al HM, Wang G, Goodman ZD. Calcifying nested stromalepithelial tumors of the liver: a clinicopathologic, immunohistochemical, and molecular genetic study of 9 cases with a longterm follow-up. Am J Surg Pathol 2009; 33(7):976-983.

5. Schaffer LR, Shehata BM, Yin J, et al. Calcifying nested stromal-epithelial tumor (CNSET) of the liver: a newly recognized entity to be considered in the radiologist's differential diagnosis. Clin Imaging 2016; 40(1):137-139.
Surgery seems to be the gold standard for therapy. In regards to chemotherapy, there are no well-defined protocols that can be applied, which suggests an individualized approach to the course of treatment [7].

\section{Conclusion}

CNSET of liver is a rare primary tumor of non-hepatic and non-biliary origins that is considered a low-grade malignancy. The diagnosis of CNSET should be established on morphology and IHC, especially when the patient is a young woman with an abdominal palpable mass, a history of oral contraceptive consumption and Cushingoid symptoms or a history of Beckwith-Weideman syndrome.

\section{Conflict of interest}

The authors have no conflict of interest to declare.

\section{Patient consent}

Written informed consent was obtained from the patient for publication of this case.

6. Fryer E, Chetty R. Unusual and rare tumours of the liver. Diagn Histopathol 2012; 18(10):449456.

7. Procopio F, Di Tommaso L, Armenia S, et al. Nested stromal-epithelial tumour of the liver: An unusual liver entity. World J Hepatol 2014; 6(3):155-159.

8. Grazi GL, Vetrone G, d'Errico A, et al. Nested stromal-epithelial tumor(NSET) of the liver: a case report of an extremely rare tumor. Pathol Res Pract 2010;206(4):282-286.

9. Meletani T, Cantini L, Lanese A, et al. Are liver nested stromal epithelial tumors always low aggressive? World J Gastroenterol 2017; 23(46):8248-8255.

10. Assmann G, Kappler R, Zeindl-Eberhart E, et al. $\beta$-Catenin mutations in 2 nested stromal epithelial tumors of the liver--a neoplasia with defective mesenchymal-epithelial transition. Hum Pathol 2012; 43(11):1815-1827.

11. Oviedo Ramírez MI, Bas Bernal A, Ortiz Ruiz E, et al. Desmoplastic nested spindle cell tumor of the liver in an adult. Ann Diagn Pathol 2010; 14(1):44-49 